\title{
Optimal and Robust PET Data Sinogram Restoration Based on the Response of the System
}

\author{
J. L. Herraiz ${ }^{1}$, S. España ${ }^{1}$, E. Vicente ${ }^{2}$, J. J. Vaquero ${ }^{2}$, M. Desco ${ }^{2}$, J. M. Udías ${ }^{1}$ \\ ${ }^{1}$ Dpto. Física Atómica, Molecular y Nuclear, Universidad Complutense de Madrid, Spain \\ ${ }^{2}$ Unidad de Medicina y Cirugía Experimental, Hospital GU “Gregorio Marañón”, Madrid, Spain
}

\begin{abstract}
We present an optimal and robust technique for the restoration of positron emission tomography (PET) data. It is based on an iterative deconvolution of Fourier Rebinned (FORE) sinograms employing the EM-ML algorithm regularized with MAP. The deconvolution kernel is related to the System Response Matrix (SRM) and the axial point spread function (PSF) caused by FORE. This method is able to deblur the acquired data whitout the introduction of additional noise and enhancing the quality (resolution, contrast) of the images reconstructed using FBP.
\end{abstract}

Index Terms - Positron emission tomography, Sinogram Restoration, Image reconstruction, Resolution, Noise.

\section{INTRODUCTION}

$\mathrm{T}$ he quality of PET images is often limited by the presence of noise and blurring. The image reconstruction methods that seek to reduce these effects can be divided in two classes:

I) Methods that make use of the SRM during the image reconstruction. These methods employ a model of the system to relate the unknown image intensities to the measureddegraded PET data. Based on these models, iterative techniques like EM-ML can be employed to achieve accurate image reconstructions.

II) Methods that filter sinograms removing blur and/or suppressing noise. These techniques are generally referred as sinogram restoration (SR) methods. Restored sinograms can be reconstructed by means of the filtered back projection (FBP) algorithm.

The second class of methods are computationally efficient and thus are often preferred in practical applications, particularly when dealing with large PET data sets. However, traditional sinogram filtering [1] is usually based on simplified

Manuscript received November 17, 2006. J. L. Herraiz e-mail: joaquin@nuclear.fis.ucm.es. S. España e-mail: samuel@nuclear.fis.ucm.es; E.Vicente email: evicente@mce.hggm.es, J.J.Vaquero email: juanjo@mce.hggm.es, M. Desco e-mail: desco@mce.hggm.es; J.M.Udias email: jose@nuc2.fis.ucm.es models and its application to single radial profiles only may not be optimal.

Recently, analytical deconvolution of the distortion produced by Fourier rebinning (FORE) [2] in PET sinograms has been attempted [3]. The restoration of the axial resolution obtained is promising, although iterative statistical techniques should probably outperform these results in noisy situations.

In this work, advanced sinogram restoration strategies are obtained following a regularized iterative method in $\rho, z$ planes. The deconvolution kernel used is based both on the SRM and on the axial PSF of FORE. This way, we can make use of our knowledge of the blurring effects involved in the emission and detection of the radiation in the PET scanner and FORE technique, prior to FBP reconstruction. The result is a sinogram with enhanced resolution and reduced noise that yields improved images.

\section{METHODS}

First, fully 3D PET data are rebinned into 2D data sets. This way, the number of oblique sinograms are reduced in general by a factor of $\mathrm{N}$, being $\mathrm{N}$ the total number of axial slices of the reconstructed. Therefore, reconstruction times are extremely reduced by the same factor. An example of a Fourier rebinned sinogram can be seen in figure 1.

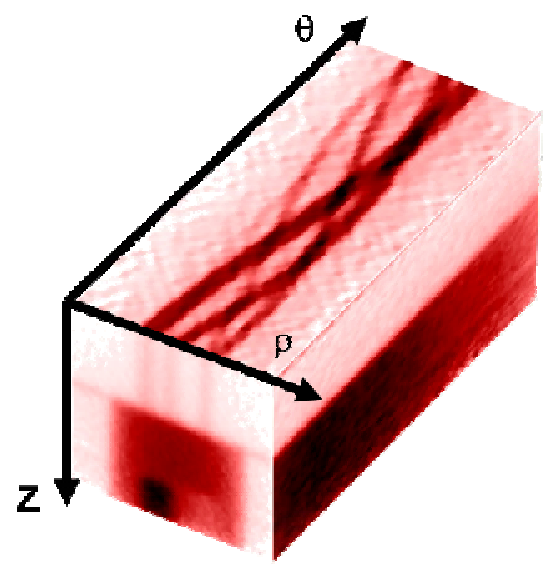

Fig.1. Fourier rebinned sinogram and $\rho, \theta, z$ coordinates 
At this point, FBP can be applied to obtain images from these rebinned data [4]. However, this approach is not optimal because, FBP being based on an idealized mathematical model of the system, it cannot account for the blur and noise in the data. Therefore, we have developed a sinogram restoration method to take into account these effects before the FBP reconstruction.

In a realistic model, the volume of space from which an emitted positron produces a coincidence count in a pair of crystals, is distributed on a wide tube of response (TOR) along the line of response (LOR) which connects them $[5,6]$.

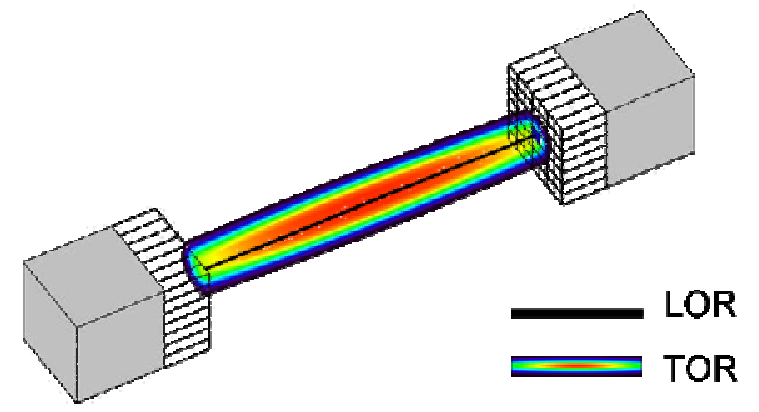

Fig. 2. Schematic representation of LOR and a TOR

In this work, we assume that the probability distribution of a TOR $h(\rho, z, t)$ can be factorized as:

$$
h(t, \rho, z) \simeq f(t) \cdot\langle g(\rho, z)\rangle
$$

$\mathrm{f}(\mathrm{t})$ represents the longitudinal profile of the TORs and $<\mathrm{g}(\rho, \mathrm{z})>$ is the average value of the transversal and transaxial profiles $g(\rho, z)$ of the TORs.

In a rotating machine like $\mathrm{PPET}$, the longitudinal profile $\mathrm{f}(\mathrm{t})$ of the TORs is quite flat and it is reasonably to neglect its impact on the acquired data. Consequently, we can approximate (1) as:

$$
h(t, \rho, z) \propto\langle g(\rho, z)\rangle
$$

The transversal and transaxial profiles $g(\rho, z)$ (Fig. 3) of the TORs cause the blurring in the data in the $\rho, z$ coordinates respectively. The 2-Dimensional distribution is equally distributed on each axis.
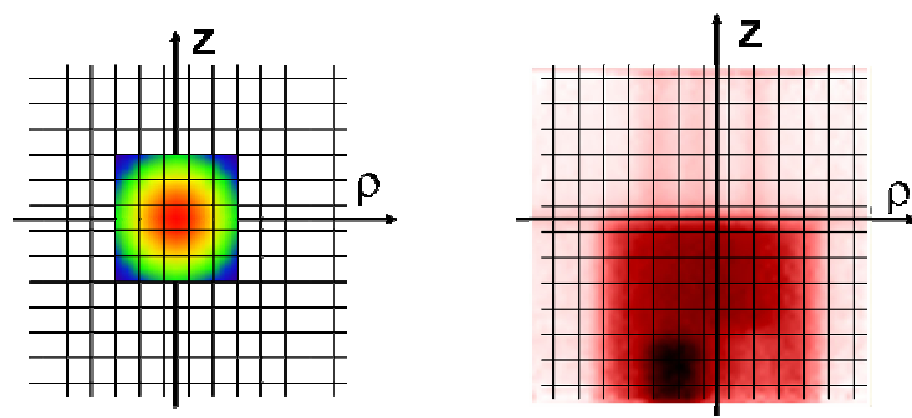

Fig. 3. Deconvolution kernel $g(\rho, z)$ and PET data in a $\rho, z$ plane
In addition to this, FORE is also known to cause blurring [3], specially in the axial (z) direction. This effect can be also included in the deconvolution kernel.

To obtain the axial PSF of FORE (PSF(Z)), a point source placed at different locations inside the field-of-view (FOV) of the scanner was projected using realistic and accurate probabilities. A new Monte Carlo code (PeneloPET) [7] that includes the main relevant physical effects was used for these projections. After that, the profiles of the data along the axial direction before using FORE (only with direct sinograms) and after applying FORE were compared. The increasing size of the profile in the $\mathrm{z}$ direction when FORE is employed is what we have called the $\mathrm{PSF}(\mathrm{Z})$ of FORE.

To take this $\operatorname{PSF}(Z)$ into account in our restoration of the sinogram, the kernel $g(\rho, z)$ was convolved $(\otimes)$ with this PSF:

$$
g^{\prime}(\rho, z)=g(\rho, z) \otimes P S F(Z)
$$

Deblur and denoise of each $\rho, z$ plane is performed via the EM-ML + MAP OSL algorithm. EM-ML can be regarded as a general tool for deconvolving images subject to non-negativity constraints [8]. MAP OSL [9] regularizes the method, making it more robust. A general formulation of the problem is:

$$
X_{j}^{n+1}=X_{j}^{n} \cdot \frac{\sum_{i=1}^{I} C_{i j} \cdot \frac{Y_{i}}{\sum_{i=1}^{I} C_{i j} \cdot X_{j}^{n}}}{\sum_{i=1}^{I} C_{i j} \cdot\left(1+\beta \frac{X_{j}^{n}-m_{j}}{m_{j}}\right)}
$$

In this equation, $X_{j}^{n}$ is the estimation of the activity of LOR $\mathrm{j}$ at the iteration $\mathrm{n}$ in the restored sinogram, $\mathrm{Y}_{\mathrm{i}}$ represents the value of the acquired data at LOR $i$, and $C_{i j}$ in our case corresponds to the deconvolution kernel g' $(\rho, z)$. MAP was implemented using the median root prior, being $\mathrm{m}_{\mathrm{j}}$ the median of the pixels in the $3 \times 3$ neightborhood of $X_{j}$ and $\beta$ the weight of the prior.

One of the main advantages of this method compared with fully $3 \mathrm{~d}$ reconstruction is that convergence is reached in only a few iterations and with a very low computational cost. This is so because in this case, the kernel is much smaller and the problem is less ill-posed. Note that no accelerated version of the EM-ML algorithm, like OS-EM, is employed here.

We have called this method, Optimal Sinogram Restoration (OSR) because it allows to incorporate the relevant information of the SRM as well as the blurring impact of FORE in the data along the z-axis during the process of sinogram restoration in a very efficient way. Having chosen a regularized version of the EM-ML algorithm, the method is robust and suitable for its application in real studies.

\section{RESULTS}

The method has been validated against real data acquired 
with rPET [10], a dedicated small animal PET scanner based on two pair of rotating planar detectors.

Acquisitions were reconstructed with FORE+FBP and compared with the proposed method, which includes sinogram restoration techniques before FBP (FORE+OSR+FBP). For the FBP reconstruction, the projections were filtered using a ramp filter multiplied by a Hanning one rolled off at the Nyquist frequency. The back-projection was pixel driven with linear interpolation performed on the projection values.

We present results from an acquisition of 200 minutes corresponding to a Hot Derenzo Phantom filled with $400 \mu \mathrm{Ci}$ of FDG. Fourier rebinned sinograms are shown in Fig. 5 (top). If we deconvolve these sinograms with the proposed method, we can yield sinograms with enhanced resolution (Fig. 5 bottom). After that, both sinograms were reconstructed using FBP with the same parameters. A transversal view of the resulting images and a profile across the line shown is depicted in Fig. 6.

The method was also applied to an acquisition of a $300 \mathrm{~g}$ rat. It was acquired during 60 minutes after being injected $2 \mathrm{mCi}$ of FDG. Figures 7 and 8 show that OSR can also be incorporated with success between FORE and FBP reconstructions in preclinical studies.

Table 1 shows a comparative study of the resolution achieved and time required for the reconstruction of an acquisition in 1CPU (Pentium IV, $2.8 \mathrm{GHz}$ ) for different methods. The use of OSR improves significantly the quality of the images with almost no additional time.

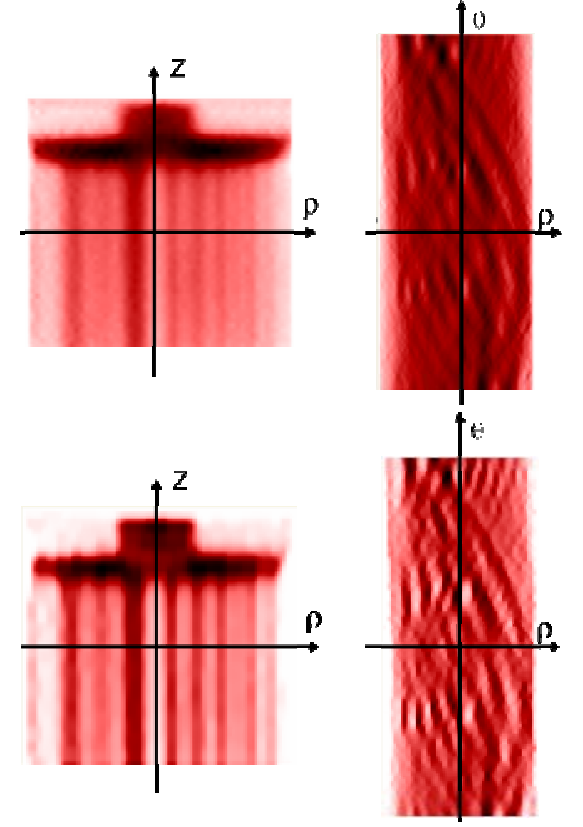

Fig 5. Results from an acquisition (200 minutes) of a Hot Derenzo Phantom filled with $400 \mu \mathrm{Ci}$ of FDG.
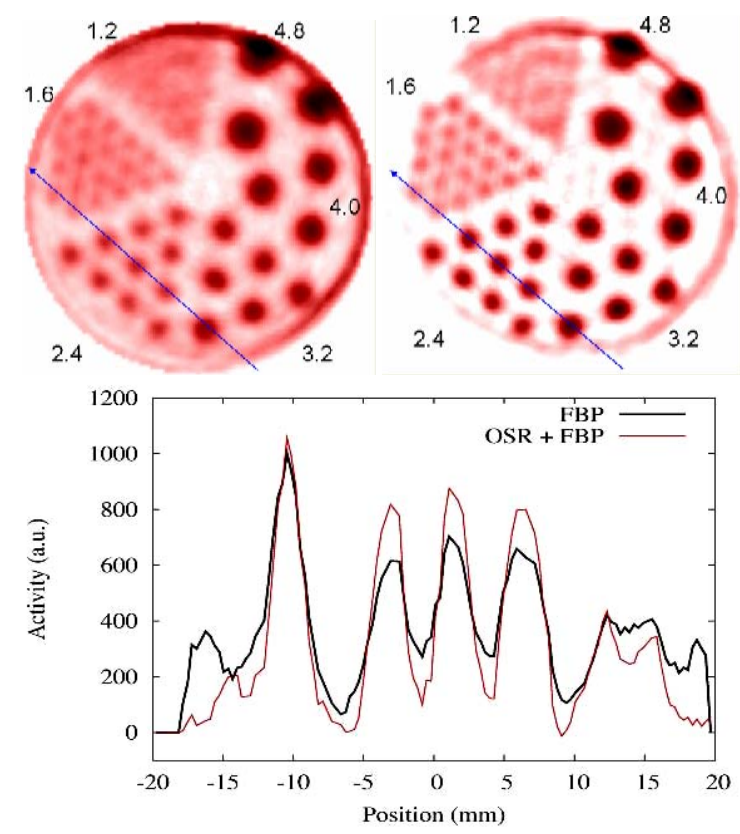

Fig 6. Reconstructed images with FBP from the data in fig. 5 and profile along the images. The diameter of each capilar is shown in $\mathrm{mm}$.

\begin{tabular}{|c|c|c|}
\hline & RESOLUTION & $\begin{array}{c}\text { RECONSTRUCTION } \\
\text { TIME }\end{array}$ \\
\hline $\begin{array}{c}\text { FORE + } \\
\text { FBP }\end{array}$ & $1.8 \mathrm{~mm}$ & $15^{\prime \prime}$ \\
\hline $\begin{array}{c}\text { FORE + } \\
\text { OSEM2D }\end{array}$ & $1.2 \mathrm{~mm}$ & $90 "$ \\
\hline $\begin{array}{c}\text { FORE + } \\
\text { OSR + FBP }\end{array}$ & $1.3 \mathrm{~mm}$ & $20 "$ \\
\hline
\end{tabular}

TABLE 1 - Resolution and CPU time required for different reconstruction methods.

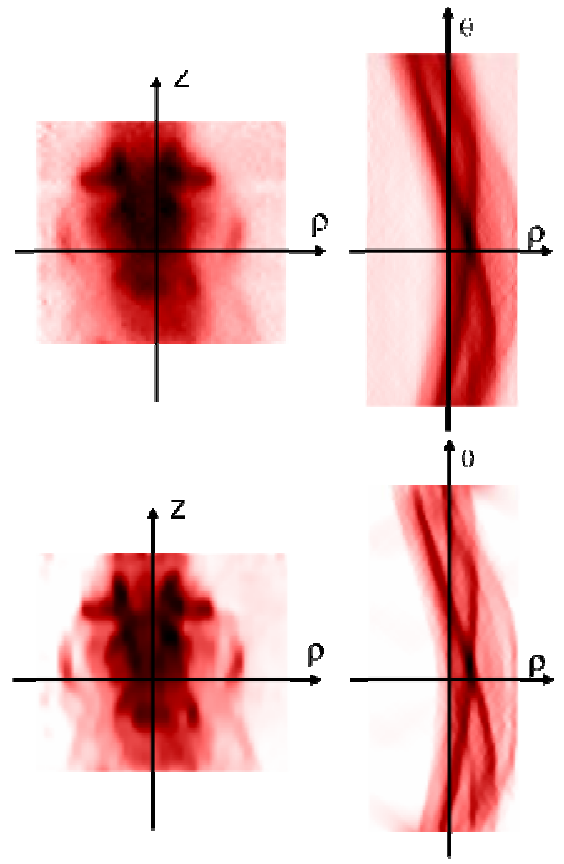

Fig 7. Results from a study of a $300 \mathrm{~g}$ rat acquired during 60 minutes after being injected $2 \mathrm{mCi}$ of FDG. Top: Sinograms before restoration. Bottom: Restored sinograms. 

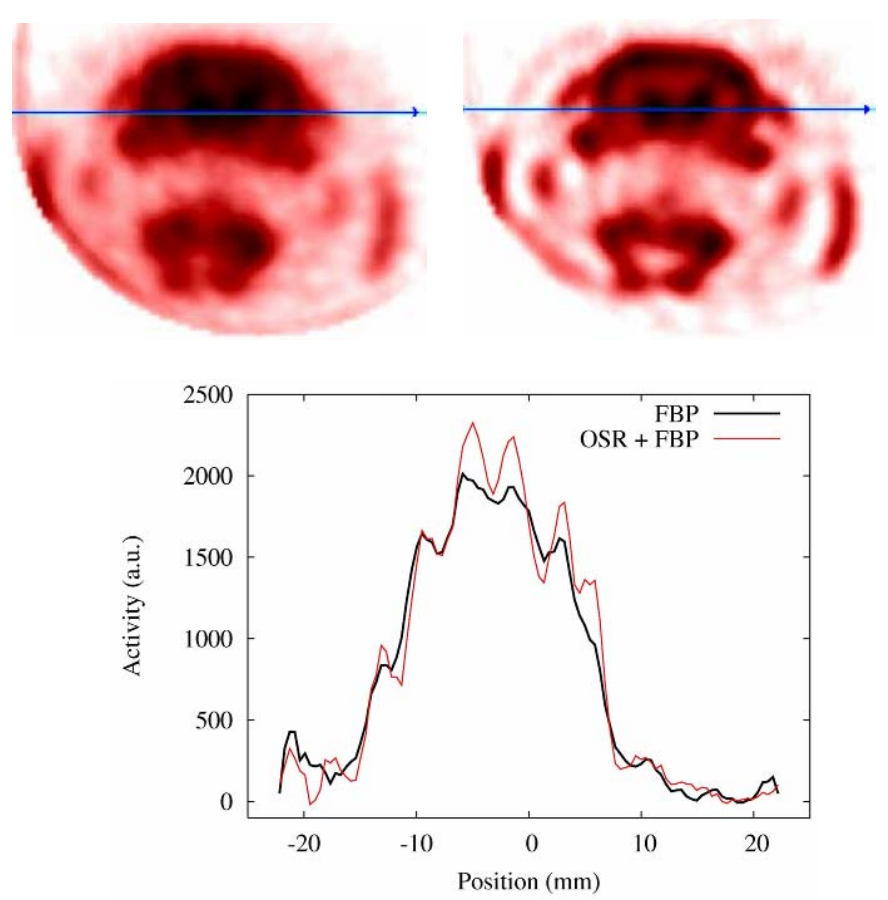

Fig 8. Reconstructed images with FBP from the data in fig. 7. Below: Profile along the images.

\section{CONCLUSIONS}

Deconvolution and denoising of the acquired PET data using iterative plus analytical methods prior to FBP reconstruction of the image can increase the resolution by more than a $30 \%$.

This sinogram restoration method is based on a realistic model of positron emission and radiation detection in the PET scanner (the System Response Matrix) and it is applied in 2dimensional planes. In this way, we can employ the information contained in the SRM while performing analytical FBP-like reconstructions and combine the benefits of fast processing of FBP with the high deblurring capabilities of iterative, SRM-based, image reconstructions.

The improvement on the quality of the reconstructed images without a significant increasing of the computational time is very promising.

\section{ACKNOWLEDGMENTS}

J.L. Herraiz acknowledges support from UCM grant. J.M. Udias acknowledges support from MEC (FPA200607393) and CENIT (Ministerio de Industria, Spain). Part of the computations of this work were done at the "High capacity cluster for physical techniques" of the Faculty for Physical Sciences of UCM, funded in part by the UE under the FEDER program and in part by the UCM.

\section{REFERENCES}

[1]

3]

4]
J. M. Udias, "FIRST: Fast Iterative Reconstruction Software for (PET) tomography," Phys. Med. Biol., vol. 51, pp. 4547, 2006. J. M. Udias, "PeneloPET: A MonteCarlo PET simulation tool based on PENELOPE," in Proc. IEEE Nuclear Science Symposium and Medical Imaging Conference, 2006.

D. L. Snyder, T. J. Schulz, and J. A. O'Sullivan, "Deblurring subject to nonnegativity constraints," IEEE Trans. Sign. Proc., vol. 40, pp. 1143-1150, 1992.

V. Bettinardi, E. Pagani, M. C. Gilardi, S. Alenius, K. Thielemans, M. Teras, and F. Fazio, "Implementation and evaluation of a 3D one-step late reconstruction algorithm for $3 \mathrm{D}$ positron emission tomography brain studies using median root prior," Eur J Nucl Med Mol Imaging, vol. 29, pp. 7-18, 2002.

J. J. Vaquero, E. Lage, L. Ricon, M. Abella, E. Vicente, and M. Desco, "rPET Detectors Design and Data Processing," in Proc. IEEE Nuclear Science Symposium and Medical Imaging Conference, 2005. 